\title{
Minimum Output Variance Control for FSN Models: Continuous-Time Case
}

\author{
GUOJUN SHI ${ }^{a}$, ROBERT E. SKELTON ${ }^{b}$ and \\ KAROLOS M. GRIGORIADIS ${ }^{\mathrm{c},}$ \\ a GM Powertrain, General Motors Corporation, 3300 GM Road, \\ MC 483-331-500, Milford, MI 48380, USA; 'b AMES Department-0411, \\ 9500 Gilman Dr, University of California at San Diego, La Jolla, \\ CA 92093-0411, USA; ' Department of Mechanical Engineering, \\ 4800 Calhoun Road, University of Houston, Houston, TX 77204-4792, USA
}

(Received 14 December 1998)

In this paper we consider the Finite Signal-to-Noise ratio model for linear stochastic systems. It is assumed that the intensity of noise corrupting a signal is proportional to the variance of the signal. Hence, the signal-to-noise ratio of each sensor and actuator is finite - as opposed to the infinite signal-to-noise ratio assumed in LQG theory. Computational errors in the controller implementation are treated similarly. The objective is to design a state feedback control law such that the closed loop system is mean square asymptotically stable and the output variance is minimized. The main result is a controller which achieves its maximal accuracy with finite control gains - as opposed to the infinite controls required to achieve maximal accuracy in LQG controllers. Necessary and sufficient conditions for optimality are derived. An optimal control law which involves the positive definite solution of a Riccati-like equation is derived. An algorithm for solving the Riccati-like equation is given and its convergence is guaranteed if a solution exists.

Keywords: Linear systems; Optimal control

\section{INTRODUCTION}

In the LQG control theory the common model for noise signals is additive noise, where the noise intensities are independent of the

\footnotetext{
* Corresponding author. Tel.: 713-743-4387. Fax: 713-743-4503.

E-mail: karolos@uh.edu.
} 
signals. In other words, the noise intensities are assumed to be constant when the system is running. This is unrealistic in practice. For instance, if a power supply is amplifying a signal which is corrupted by an external noise, it also amplifies the noise. The same is true for electromechanical devices. In aerospace engineering, spacecraft are often rotated about their symmetry axis in order to enhance their aerodynamic stability upon reentry. Subjecting the constant angular rate of the symmetry axis to a perturbation results in a disturbance that depends on the precession rates. Thus the assumption that the noise intensities are independent of the energy of the signal leads to several deficiencies in the practical applications of the LQG theory, because the real sensor and actuator devices have noise sources that are related to the strength of the signal part. In this paper we consider the finite signal-to-noise ratio models for linear stochastic systems, which was introduced in [1]. It is assumed that the intensity of the noise is related to the signal covariance in proportion to the noise-to-signal ratio. The same assumption is made concerning computational errors in controller implementation, since large signals suffer larger computational errors. For detailed descriptions, see [2-4].

We seek to minimize the variance of the system output $y_{p}$. Our new model and controller are still linear, but with finite signal-to-noise ratios in the hardware and software devices. The cost function is still quadratic in the output. We will call the new model the finite signalto-noise (FSN) model of linear systems, the new controllers FSN controllers.

In optimal control problems, we will demonstrate that these more realistic noise assumptions will keep the control variance relatively small, since a very large control variance comes at the price of very large additive noise, which degrades performance. Hence, in the new theory there is a self-correcting mechanism to keep the control finite in the output variance minimization problem, as opposed to LQG theory, where minimum output variance occurs at infinite control gain. This can have a significant impact on robustness of the controller, since larger control signals have a greater tendency to destabilize unmodeled dynamics in the system, due to "control spillover". Other engineering benefits of the FSN model and the new controller will be 
discussed in the sequel.

- This allows the cost function to have physical significance: Of course, each of the two terms, i.e., the output and control variances, in the cost function of LQG have physical significance, but their sum is not a physical entity. Using a non-physical scalar cost function is an artificial "device" used for design. Choosing the LQG weights is a non-trivial task $[5,6]$. The new theory treats physically meaningful cost functions (output variance). Weights on the output still must be selected, but weights on control can be zero.

- The new theory accommodates the singular case: Setting control penalties to zero in LQG complicates the theory since maximal accuracy occurs at infinite gain (for continuous-time case). Singular solutions are not obtained simply by setting weights to zero in the nominal theory. The new theory accommodates the singular case without modifications.

- Maximal accuracy occurs with a finite control: In LQG, the maximal accuracy of the output variance occurs at infinite control effort. Hence some weighting on control in the cost function is necessary for practical implementation. The FSN problem yields a maximal accuracy with finite control effort, hence no weighting on the control signals is required to make the optimization problem well posed.

- Finite precision controller: LQG controllers can perform poorly in a finite precision implementation. In fact, the computational errors can dominate the performance [7,8]. The FSN controller is optimized with computational errors modeled as white noise with finite signal-to-noise ratios.

All of the above "engineering" improvements over LQG are made possible by the addition of signal dependent noise added to the system model. This is the key difference with LQG. The price we must pay for this improvement is a mild increase in computational complexity. The separation theorem does not hold. The LQG theory is very complete, given the standard modeling assumptions. It is our opinion, however, that many of the practical deficiencies of LQG theory [9] can be traced to the presumption that the signal-to-noise ratio is infinite in the acuators, sensors, and controller computations. 
The control problems of FSN models were attacked in [2-4]. In $[2,4]$ the covariance control LMI approach was used. In [3] the $\mu_{2}$ measure was introduced and a $D-K$ iteration approach was used. In this paper, our attention is focused on the state feedback case. The objective is to design an optimal state feedback controller in the sense that the closed loop system is mean square asymptotically (MSA) stable and produces the minimum output variance. Based on a Riccati-like equation, necessary and sufficient conditions for optimality are provided. An algorithm for solving the Riccati-like equation is suggested and the convergence is guaranteed if the FSN system is MSA stabilizable. It should be noticed that $[10,11]$ used different model concepts to get a similar optimal controller.

The remainder of the paper is organized as follows. In the next section we introduce the FSN model for linear stochastic systems and also formulate the minimum output variance control problem. The minimum output variance control problem is solved in terms of a Riccati-like equation in Section 3. Section 4 provides an algorithm for solving the Riccati-like equation and the convergence property of the algorithm. In Section 5, a numerical example is given to illustrate the theory. Finally, conclusions are made in Section 6.

The notation used in this paper is fairly standard. Matrices are denoted by italic letters. For a matrix $A, A^{\mathrm{T}}$ denotes its transpose. The trace of a square matrix $S$ is denoted $\operatorname{tr} S$. For symmetric matrices $P$ and $Q, P>0(P \geq 0)$ denotes the fact that $P$ is positive definite (positive semidefinite), $P>Q(P \geq Q)$ denotes $P-Q>0(P-Q \geq 0)$. The notation $\|P\|$ denotes the spectral norm of the matrix $P$. For a vector $x,\|x\|$ denotes the usual Euclidean norm of $x . \mathcal{E}\{\cdot\}$ denotes the expectation operator and $\mathcal{E}_{\infty}\{\cdot\}$ denotes $\lim _{t \rightarrow \infty} \mathcal{E}\{\cdot\}$. vec $\{\cdot\}$ denotes the column-stacking operator. $\otimes$ denotes the Kronecker product.

\section{FSN MODEL AND PROBLEM FORMULATION}

Consider the block diagram of a linear system depicted in Fig. 1. This figure describes the structure of our model, which includes six zeromean white noise sources, $w_{a}, w_{p}, w_{s}, w_{z}, w_{u}$, and $w_{c}$. Three sources $w_{a}$, $w_{p}, w_{s}$ are associated with the plant and its actuating-sensing hardware. The sources $w_{z}, w_{c}$ and $w_{u}$ are computational errors associated with 


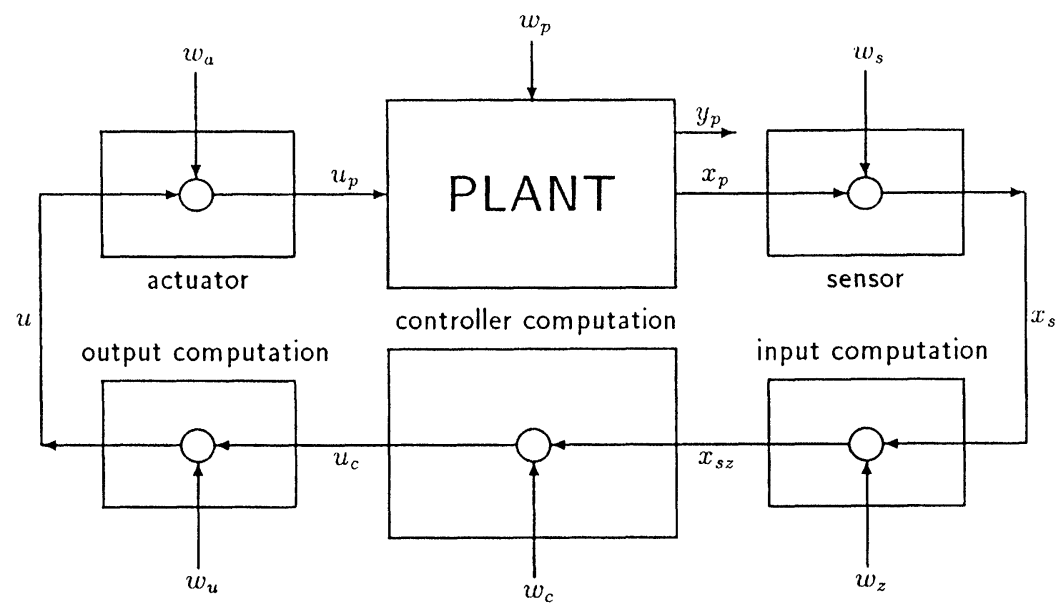

FIGURE 1 State feedback control for FSN linear systems.

controller implementation. In a digital implementation, $w_{z}$ would be quantization error in the $\mathrm{A} / \mathrm{D}$ converter, $w_{c}$ would be the roundoff errors in the controller gain multiplication, $w_{u}$ would be the quantization error in the $\mathrm{D} / \mathrm{A}$ converter. In the analog computations of continuous-time systems $w_{z}, w_{c}$ and $w_{u}$ are noise sources from the operational amplifiers performing the calculations in the analog computer. It is more realistic to assume that the intensity of each noise increases with the size (variance) of the associated uncorrupted signal. Consider the white noise $w_{a}$. For our purposes, we let $w_{a}=w_{a 1}+w_{a 2}$, where $w_{a 1}$ and $w_{a 2}$ are independent white noise sources, $w_{a 1}$ is the ambient noise, modeled by the traditional assumptions, and $w_{a 2}$ is related to the signal-to-noise ratio (SNR) of the actuator device, defined as follows.

$$
\begin{aligned}
& \mathcal{E}_{\infty}\left\{w_{a}(t)\right\}=0, \\
& \mathcal{E}_{\infty}\left\{w_{a}(t) w_{a}^{\mathrm{T}}(\tau)\right\}=W_{a} \delta(t-\tau)=\left(W_{a 1}+W_{a 2}\right) \delta(t-\tau),
\end{aligned}
$$

where

$$
W_{a 1}=\text { constant }, \quad W_{a 2}=\mathcal{C}\left\{\Sigma_{a} u(t)\right\}, \Sigma_{a}=\operatorname{diag}\left\{\cdots \sigma_{a_{i}} \cdots\right\},
$$


where $\mathcal{C}\{\cdot\}$ is a special operator denoting covariance of the signal part of vector $\{\cdot\}$, and $\sigma_{a_{i}}^{-1}$ is the SNR for the $i$ th actuator. The inverse of the SNR will be called the noise-to-signal ratio (NSR). All noises are uncorrelated with the initial plant state $x_{p}(0)$, which is zero mean. The NSR of all hardware and computational devices enters into the model by relating the intensity of the noise source to the covariance of the signal as in (2.2). For simplicity, we assume that, in one hardware device, all of the channels have the same signal-to-noise ratio, e.g., $\Sigma_{a}=\sigma_{a} I$.

In Fig. 1, all of the signals are related by

$$
\begin{aligned}
\dot{x}_{p} & =A_{p} x_{p}+B_{p} u_{p}+w_{p}, \\
y_{p} & =C_{p} x_{p}, \\
u_{p} & =u+w_{a}, \\
x_{s} & =x_{p}+w_{s}, \\
x_{s z} & =x_{s}+w_{z}, \\
u & =u_{c}+w_{u}, \\
u_{c} & =G x_{s z}+w_{c},
\end{aligned}
$$

where $x_{p} \in \Re^{n_{p}}$ is the plant state, $u_{p} \in \Re^{n_{u}}$ is the control signal to the plant, $y_{p} \in \Re^{n_{y}}$ is the output to be regulated, $x_{s} \in \Re^{n_{p}}$ is the measurement of the states, $x_{s z} \in \Re^{n_{p}}, u \in \Re^{n_{u}}, u_{c} \in \Re^{n_{u}}$ are the signals indicated in Fig. $1, A_{p}, B_{p}$ and $C_{p}$ are real constant matrices with appropriate dimensions, $G \in \Re^{n_{u} \times n_{x}}$ is the controller gain to be designed, and the noises

$$
\begin{aligned}
& w_{p}=D_{p} w_{p 1}+w_{p 2}, \\
& w_{a}=w_{a 1}+w_{a 2}, \\
& w_{s}=w_{s 1}+w_{s 2}, \\
& w_{z}=w_{z 1}+w_{z 2}, \\
& w_{c}=w_{c 1}+w_{c 2}, \\
& w_{u}=w_{u 1}+w_{u 2}
\end{aligned}
$$

are zero-mean white noises. For $k=p, a, s, z, c, u$, the intensity of $w_{k 1}$, is $W_{k 1}>0$, the intensities of $w_{k 2}, W_{k 2}$, will be given in the following. It is assumed in the state feedback case of this paper that $w_{s 1}=0, w_{z 1}=0$. 
Now one can obtain the closed loop system from the Eqs. in (2.3)

$$
\begin{aligned}
& \dot{x}_{p}=\left(A_{p}+B_{p} G\right) x_{p}+w, \\
& y_{p}=C_{p} x
\end{aligned}
$$

where

$$
w \triangleq B_{p}\left(w_{a}+w_{u}+w_{c}+G w_{s}+G w_{z}\right)+w_{p}
$$

Suppose the state covariance of the closed loop system, $X_{p} \triangleq$ $\mathcal{E}_{\infty}\left\{x(t) x^{\mathrm{T}}(t)\right\}$, exists. Then using the operator $\mathcal{C}\{\cdot\}$, one can show that

$$
\begin{aligned}
W_{a 2} & =\mathcal{C}\left\{\sigma_{a} u(t)\right\} \\
& =\sigma_{a}^{2} \mathcal{C}\left\{G\left(x_{p}+w_{s}+w_{z}\right)+w_{c}+w_{u}\right\} \\
& =\sigma_{a}^{2} \mathcal{E}_{\infty}\left\{G x_{p}\right\} \\
& =\sigma_{a}^{2} G X_{p} G^{\mathrm{T}} .
\end{aligned}
$$

Similarly,

$$
\begin{aligned}
& W_{u 2}=\mathcal{C}\left\{\sigma_{u} u_{c}(t)\right\}=\sigma_{u}^{2} G X_{p} G^{\mathrm{T}}, \\
& W_{p 2}=\mathcal{C}\left\{\sigma_{p} x_{p}(t)\right\}=\sigma_{p}^{2} X_{p}, \\
& W_{s 2}=\mathcal{C}\left\{\sigma_{s} x_{p}(t)\right\}=\sigma_{s}^{2} X_{p}, \\
& W_{z 2}=\mathcal{C}\left\{\sigma_{z} x_{s}(t)\right\}=\sigma_{z}^{2} X_{p}, \\
& W_{c 2}=\mathcal{C}\left\{\sigma_{c} G x_{s z}(t)\right\}=\sigma_{c}^{2} G X_{p} G^{\mathrm{T}} .
\end{aligned}
$$

Hence, the intensity of the white noise $w$ in (2.5) is given by

$$
W\left(X_{p}, G\right)=\sigma^{2} B_{p} G X_{p} G^{\mathrm{T}} B_{p}^{\mathrm{T}}+\sigma_{p}^{2} X_{p}+B_{p} W_{\mathrm{auc}} B_{p}^{\mathrm{T}}+D_{p} W_{p 1} D_{p}^{\mathrm{T}},
$$

where

$$
W_{\mathrm{auc}} \triangleq W_{a 1}+W_{u 1}+W_{c 1}, \quad \sigma^{2} \triangleq \sigma_{a}^{2}+\sigma_{c}^{2}+\sigma_{s}^{2}+\sigma_{z}^{2}+\sigma_{u}^{2} .
$$

The scalar $I_{Q} \triangleq \sigma^{-2}$ is referred to as the INFORMATION QUALITY of the FSN system. Consider the closed-loop system (2.3). It can be 
shown that the state covariance $X_{p}$ satisfies the following Lyapunov equation

$$
\left(A_{p}+B_{p} G\right) X_{p}+X_{p}\left(A_{p}+B_{p} G\right)^{\mathrm{T}}+W\left(X_{p}, G\right)=0 .
$$

This matrix equation is not the conventional Lyapunov equation since $W\left(X_{p}, G\right)$ is a function of covariance $X_{p}$. Substituting for $W\left(X_{p}, G\right)$ into the Eq. (2.11) and collecting terms, we have a Lyapunov-like equation

$$
\begin{aligned}
\mathcal{L}\left(X_{p}, G\right) \triangleq & \left(A_{o}+B_{p} G\right) X_{p}+X_{p}\left(A_{o}+B_{p} G\right)^{\mathrm{T}}+\sigma^{2} B_{p} G X_{p} G^{\mathrm{T}} B_{p}^{\mathrm{T}} \\
& +B_{p} W_{\mathrm{auc}} B_{p}^{\mathrm{T}}+D_{p} W_{p 1} D_{p}^{\mathrm{T}}=0
\end{aligned}
$$

where $A_{o} \triangleq A_{p}+\frac{1}{2} \sigma_{p}^{2} I_{n_{p}}$.

We shall make the following assumptions about the FSN model:

ASSUMPTION 2.1

(i) The pair $\left(A_{p}, B_{p}\right)$ is controllable;

(ii) The pair $\left(C_{p}, A_{p}\right)$ is observable;

(iii) The input matrix $B_{p}$ has full column rank.

DEFINITION 2.1 The FSN closed loop system is MSA stable if its steady state covariance $X_{p}$ exists and is positive definite.

DEFINITION 2.2 The FSN open loop system is MSA stabilizable if there exists a gain $G$ such that the FSN closed loop system is MSA stable.

In this paper it is also assumed that a noisy measurement of the state of the FSN system is available for feedback. We shall consider the following problem.

Minimum Output Variance Control Problem Consider the FSN system (2.3). Find a MSA stabilizing state feedback controller such that the output variance $\operatorname{tr}\left[C_{p} X_{p} C_{p}^{\mathrm{T}}\right]$ is minimized subject to (2.12).

The following results will be used in the proof of theorems.

Lemma 1 [12] Suppose the pair $(A, B)$ is stabilizable and the Riccati equation

$$
A^{\mathrm{T}} X_{1}+X_{1} A-X_{1} B B^{\mathrm{T}} X_{1}+Q_{1}=0
$$


has a symmetric solution $X_{1}$. Furthermore, suppose $Q_{2} \geq Q_{1}$. Then the Riccati equation

$$
A^{\mathrm{T}} X_{2}+X_{2} A-X_{2} B B^{\mathrm{T}} X_{2}+Q_{2}=0
$$

has a unique strong solution ${ }^{\dagger} X_{2} \geq X_{1}$.

LEMma 2 [12] Suppose the Riccati equation

$$
A^{\mathrm{T}} X_{1}+X_{1} A-X_{1} M_{1} X_{1}+Q=0
$$

has a symmetric solution $X_{1}$. Furthermore, suppose $M_{1} \geq M_{2} \geq 0$ where $\left(A, M_{2}\right)$ is stabilizable. Then the Riccati equation

$$
A^{\mathrm{T}} X_{2}+X_{2} A-X_{2} M_{2} X_{2}+Q=0
$$

has a unique strong solution $X_{2} \geq X_{1}$.

LEMMA 3 [13, pp. 186-199] Let $\left\{P_{i}: i=1,2, \ldots\right\}$ be a sequence of $n \times n$ symmetric matrices such that $P_{1} \leq P_{2} \leq \cdots$. Suppose that there exists a symmetric matrix $P$ such that $P_{i} \leq P, i=1,2, \ldots$ Then $P_{\infty}=\lim _{i \rightarrow \infty} P_{i}$ exists and $P_{\infty} \leq P$.

The lemma is a special case of a result for positive operators in Hilbert space. The result is also true for a monotone decreasing sequence which is bounded below.

\section{MINIMUM OUTPUT VARIANCE CONTROLLER DESIGN}

In this section we shall solve the minimum output variance control problem for the FSN systems and reveal some features of the optimal controller.

Proposition 3.1 Consider the FSN system and let a controller gain $G$ be given. Then the following statements are equivalent:

(i) The FSN system is MSA stabilized by G;

(ii) The matrix

$$
\mathcal{A} \triangleq I_{n_{p}} \otimes\left(A_{o}+B_{p} G\right)+\left(A_{o}+B_{p} G\right) \otimes I_{n_{p}}+\sigma^{2}\left(B_{p} G\right) \otimes\left(B_{p} G\right)
$$

is stable;

\footnotetext{
${ }^{\dagger}$ The matrix $A-B B^{\mathrm{T}} X_{2}$ has no eigenvalues in the open right half plane.
} 
(iii) The Lyapunov-like equation (2.12) has a solution $X_{p}>0$;

(iv) The dual Lyapunov-like equation

$$
\left(A_{o}+B_{p} G\right)^{\mathrm{T}} Y_{p}+Y_{p}\left(A_{o}+B_{p} G\right)+\sigma^{2} G^{\mathrm{T}} B_{p}^{\mathrm{T}} Y_{p} B_{p} G+C_{p}^{\mathrm{T}} C_{p}=0
$$

has a solution $Y_{p}>0$.

Proof The equivalence of (i) and (iii) follows from Definitions 2.1 and 2.2. For the equivalence of (ii), (iii) and (iv), see [10,14].

The following theorem is our main result, which provides the minimum output variance optimal controller for the FSN systems.

THEOREM 1 The minimum output variance control problem has a solution if and only if the Riccati-like equation

$$
A_{o}^{\mathrm{T}} Y_{p}+Y_{p} A_{o}-\frac{1}{\sigma^{2}} Y_{p} B_{p}\left(B_{p}^{\mathrm{T}} Y_{p} B_{p}\right)^{-1} B_{p}^{\mathrm{T}} Y_{p}+C_{p}^{\mathrm{T}} C_{p}=0
$$

has a solution $Y_{p}>0$, where $A_{o}=A_{p}+\frac{1}{2} \sigma_{p}^{2} I_{n_{p}}$. In this case, the FSN optimal controller is given by

$$
G=-\frac{1}{\sigma^{2}}\left(B_{p}^{\mathrm{T}} Y_{p} B_{p}\right)^{-1} B_{p}^{\mathrm{T}} Y_{p}
$$

the minimum output variance

$$
J_{y}\left(Y_{p}\right)=\operatorname{tr}\left[Y_{p}\left(B_{p} W_{\mathrm{auc}} B_{p}^{\mathrm{T}}+D_{p} W_{p 1} D_{p}^{\mathrm{T}}\right)\right],
$$

and the control effort

$$
J_{u}\left(X_{p}, G\right)=\operatorname{tr}\left[G X_{p} G^{\mathrm{T}}\right]
$$

where $X_{p}$ is the solution of the Lyapunov-like equation (2.12).

Proof Necessity Suppose that the minimum output variance control problem has a solution. This implies that there exists a controller gain $G$ such that the closed loop system is MSA stable, i.e., the Lyapunovlike equation (2.12) has a positive definite solution $X_{p}$, and the output 
variance $\operatorname{tr}\left[C_{p} X_{p} C_{p}^{\mathrm{T}}\right]$ is minimal. Then Proposition 3.1 suggests that the matrix $\mathcal{A}$ is stable. We show the necessity by using the Lagrange Multiplier method $[15,16]$.

Let

$$
J\left(X_{p}, G\right) \triangleq \operatorname{tr}\left[C_{p} X_{p} C_{p}^{\mathrm{T}}\right]+\operatorname{tr}\left[\mathcal{L}\left(X_{p}, G\right) Y_{p}\right]
$$

with $Y_{p}=Y_{p}^{\mathrm{T}}$. We first verify the regularity conditions. Note that

$$
\begin{aligned}
& {\left[\begin{array}{l}
\frac{\partial \operatorname{tr}\left[\mathcal{L}\left(X_{p}, G\right) Y_{p}\right]}{\partial X_{p}} \\
\left.\frac{\partial \operatorname{tr}\left[\mathcal{L}\left(X_{p}, G\right) Y_{p}\right]}{\partial G}\right]
\end{array}\right.} \\
& \quad=\left[\begin{array}{c}
\left(A_{o}+B_{p} G\right)^{\mathrm{T}} Y_{p}+Y_{p}\left(A_{o}+B_{p} G\right)+\sigma^{2} G^{\mathrm{T}} B_{p}^{\mathrm{T}} Y_{p} B_{p} G \\
2\left(B_{p}^{\mathrm{T}} Y_{p}+\sigma^{2} B_{p}^{\mathrm{T}} Y_{p} B_{p} G\right) X_{p}
\end{array}\right]=0
\end{aligned}
$$

has a unique solution $Y_{p}=0$. Indeed, the first block is equivalent to

$$
\mathcal{A} \operatorname{vec} Y_{p}=0
$$

which implies that vec $Y_{p}=0$, since $\mathcal{A}$ is nonsingular. Hence regularity conditions are satisfied. We now apply the necessary conditions for local optimum:

$$
\begin{aligned}
\frac{\partial J\left(X_{p}, G\right)}{\partial X_{p}}= & \left(A_{o}+B_{p} G\right)^{\mathrm{T}} Y_{p}+Y_{p}\left(A_{o}+B_{p} G\right) \\
& +\sigma^{2} G^{\mathrm{T}} B_{p}^{\mathrm{T}} Y_{p} B_{p} G+C_{p}^{\mathrm{T}} C_{p}=0 \\
\frac{\partial J\left(X_{p}, G\right)}{\partial G}= & 2\left(B_{p}^{\mathrm{T}} Y_{p}+\sigma^{2} B_{p}^{\mathrm{T}} Y_{p} B_{p} G\right) X_{p}=0 \\
\frac{\partial J\left(X_{p}, G\right)}{\partial Y_{p}}= & \mathcal{L}\left(X_{p}, G\right)=0 .
\end{aligned}
$$

Since $X_{p}>0$, the optimal controller (3.4) follows from (3.10). We substitute this relation into Eq. (3.9) and get the Riccati-like equation (3.3). By Proposition 3.1, this Riccati-like equation has a 
solution $Y_{p}>0$. Furthermore, the minimum output variance ${ }^{\ddagger}$

$$
\begin{aligned}
\operatorname{tr}\left[C_{p} X_{p} C_{p}^{\mathrm{T}}\right] & =\left(\operatorname{vec} X_{p}\right)^{\mathrm{T}} \operatorname{vec}\left(C_{p}^{\mathrm{T}} C_{p}\right) \\
& =\left[\mathcal{A}^{-1} \operatorname{vec}\left(B_{p} W_{\mathrm{auc}} B_{p}^{\mathrm{T}}+D_{p} W_{p 1} D_{p}^{\mathrm{T}}\right)\right]^{\mathrm{T}} \operatorname{vec}\left(C_{p}^{\mathrm{T}} C_{p}\right) \\
& =\left[\operatorname{vec}\left(B_{p} W_{\mathrm{auc}} B_{p}^{\mathrm{T}}+D_{p} W_{p 1} D_{p}^{\mathrm{T}}\right)\right]^{\mathrm{T}} \mathcal{A}^{-\mathrm{T}} \operatorname{vec}\left(C_{p}^{\mathrm{T}} C_{p}\right) \\
& =\left[\operatorname{vec}\left(B_{p} W_{\mathrm{auc}} B_{p}^{\mathrm{T}}+D_{p} W_{p 1} D_{p}^{\mathrm{T}}\right)\right]^{\mathrm{T}} \operatorname{vec} Y_{p} \\
& =\operatorname{tr}\left[Y_{p}\left(B_{p} W_{\mathrm{auc}} B_{p}^{\mathrm{T}}+D_{p} W_{p 1} D_{p}^{\mathrm{T}}\right)\right]
\end{aligned}
$$

It is straightforward to show that the control effort is given by (3.6).

Sufficiency Suppose that the Riccati-like equation (3.3) has a positive definite solution $Y_{p}$. Let $G$ be given by (3.4). Then Eq. (3.3) can be rewritten as (3.2). It follows from Proposition 3.1, that the Lyapunovlike equation (2.12) has a positive definite solution $X_{p}$. By definition, therefore, the controller (3.4) is MSA stabilizing. Suppose $G_{a}$ is an arbitrary MSA stabilizing controller. By Proposition 3.1, the Riccatilike equation

$$
\left(A_{o}+B_{p} G_{a}\right)^{\mathrm{T}} Y_{a}+Y_{a}\left(A_{o}+B_{p} G_{a}\right)+\sigma^{2} G_{a}^{\mathrm{T}} B_{p}^{\mathrm{T}} Y_{a} B_{p} G_{a}+C_{p}^{\mathrm{T}} C_{p}=0
$$

has a solution $Y_{a}>0$. Completing square leads to

$$
\begin{aligned}
A_{o}^{\mathrm{T}} Y_{a} & +Y_{a} A_{o}-\frac{1}{\sigma^{2}} Y_{a} B_{p}\left(B_{p}^{\mathrm{T}} Y_{a} B_{p}\right)^{-1} B_{p}^{\mathrm{T}} Y_{a}+C_{p}^{\mathrm{T}} C_{p} \\
= & -\left[G_{a}+\sigma^{-2}\left(B_{p}^{\mathrm{T}} Y_{a} B_{p}\right)^{-1} B_{p}^{\mathrm{T}} Y_{a}\right]^{\mathrm{T}}\left(\sigma^{2} B_{p}^{\mathrm{T}} Y_{p} B_{p}\right) \\
& \times\left[G_{a}+\sigma^{-2}\left(B_{p}^{\mathrm{T}} Y_{a} B_{p}\right)^{-1} B_{p}^{\mathrm{T}} Y_{a}\right] \\
\leq & 0 .
\end{aligned}
$$

It follows from Lemma 1 that $0<Y_{p} \leq Y_{a}$ and hence $J_{p}\left(Y_{p}\right) \leq J_{p}\left(Y_{a}\right)$. Therefore, the controller (3.4) is the optimal solution of the minimum output variance control problem.

\footnotetext{
${ }^{\ddagger}$ An alternative proof for this is to begin with (3.7).
} 
Remark 3.1 Suppose the minimum output variance control problem has a solution. Then the nominal system matrix $A_{p}+B_{p} G$ has a stability margin $\frac{1}{2} \sigma_{p}^{2}$. That is, all eigenvalues of $A_{p}+B_{p} G$ lie to the left of the vertical line $-\frac{1}{2} \sigma_{p}^{2}+j \omega$.

The Riccati-like equation is not the standard Riccati equation and hence cannot be solved directly. In the next section, a successive approximation algorithm will be provided.

\section{SUCCESSIVE APPROXIMATION ALGORITHM}

As can be seen from Theorem 1, the minimum output control problem involves the positive solution $Y_{p}$ of the Riccati-like equation (3.3). For solving this kind of Riccati-like equation with control penalty $R \neq 0$, an iteration algorithm was proposed in [17]. For the Riccati-like equation with control penalty $R=0$, we shall give a modified algorithm.

LEMMA 4 Suppose that the Riccati-like equation (3.3) has a solution $Y_{p}>0$. Then, for an arbitrary $0<R<\infty$, the following Riccati-like equation

$$
A_{o}^{\mathrm{T}} S+S A_{o}-S B_{p}\left(R+\sigma^{2} B_{p}^{\mathrm{T}} S B_{p}\right)^{-1} B_{p}^{\mathrm{T}} S+C_{p}^{\mathrm{T}} C_{p}=0
$$

has a solution $S>Y_{p}>0$.

Lemma 4 can be considered an extension of Lemma 2. To prove Lemma 4, we recall the following algorithm [17]:

Algorithm I Let $\left(A_{p}, B_{p}, C_{p}, \sigma^{2}, \sigma_{p}^{2}\right)$ be given. Set index $i=0$ and $S_{0}=0$. Choose a prescribed tolerance $\epsilon>0$, matrix $R>0$, and the maximal iteration number $N_{\max }$. For $i \geq 0$, solve the Riccati equations

$$
\begin{gathered}
A_{o}^{\mathrm{T}} S_{i+1}+S_{i+1} A_{o}-S_{i+1} B_{p} R_{i}^{-1} B_{p}^{\mathrm{T}} S_{i+1}+C_{p}^{\mathrm{T}} C_{p}=0 \\
R_{i}=R+\sigma^{2} B_{p}^{\mathrm{T}} S_{i} B_{p}
\end{gathered}
$$

to obtain a sequence of positive definite solutions $\left\{S_{i}: i=1,2, \ldots\right\}$ until $\left\|S_{i+1}-S_{i}\right\|<\epsilon$ or $i>N_{\max }$. 
Suppose that $S \triangleq \lim _{i \rightarrow \infty} S_{i}>0$ exists. Then we propose the following algorithm for solving the Riccati-like equation (3.3).

\section{Algorithm II}

Step 0 Solve Algorithm I to obtain $S>0$.

Step 1 Set index $i=0$ and $Y_{p}^{0}=S$. Choose a prescribed tolerance $\epsilon>0$ and the maximal iteration number $N_{\max }$. For $i \geq 0$, solve the Riccati equations

$$
A_{o}^{\mathrm{T}} Y_{p}^{i+1}+Y_{p}^{i+1} A_{o}-Y_{p}^{i+1} B_{p}\left(\sigma^{2} B_{p}^{\mathrm{T}} Y_{p}^{i} B_{p}\right)^{-1} B_{p}^{\mathrm{T}} Y_{p}^{i+1}+C_{p}^{\mathrm{T}} C_{p}=0
$$

to obtain a sequence of positive definite solutions $\left\{Y_{p}^{i}: i=1,2, \ldots\right\}$ until $\left\|Y_{p}^{i+1}-Y_{p}^{i}\right\|<\epsilon$ or $i>N_{\max }$.

Proof of Lemma 4 By the assumption that $\left(A_{p}, B_{p}, C_{p}\right)$ is controllable and observable, one can show that $\left(A_{o}, B_{p}, C_{p}\right)$ is controllable and observable [18]. Now suppose that there exists a solution $Y_{p}>0$ to the Riccati-like equation (3.3). For arbitrary $R>0$, we first show by construction that there exists a solution $S>0$ to the Eq. (4.1). Consider Algorithm I. Notice that $\left\{S_{i}: i=1,2, \ldots\right\}$ is a monotonically nondecreasing sequence. It suffices to show that this sequence is bounded above.

Let $G=\left(1 / \sigma^{2}\right)\left(B_{p}^{\mathrm{T}} Y_{p} B_{p}\right)^{-1} B_{p}^{\mathrm{T}} Y_{p}$. Then the Eq. (3.3) can be rewritten as

$$
\left(A_{o}+B_{p} G\right)^{\mathrm{T}} Y_{p}+Y_{p}\left(A_{o}+B_{p} G\right)+\sigma^{2} G^{\mathrm{T}} B_{p}^{\mathrm{T}} Y_{p} B_{p} G+C_{p}^{\mathrm{T}} C_{p}=0 .
$$

The matrix $A_{\diamond}+B_{p} G$ is stable. It follows from Lyapunov theory that the following equation

$$
\left(A_{o}+B_{p} G\right)^{\mathrm{T}} \bar{S}+\bar{S}\left(A_{o}+B_{p} G\right)+\sigma^{2} G^{\mathrm{T}} B_{p}^{\mathrm{T}} \bar{S} B_{p} G+G^{\mathrm{T}} R G+C_{p}^{\mathrm{T}} C_{p}=0 .
$$

has a solution $\bar{S}>0$ for any $R>0$. On the other hand, the Riccati equation (4.2) can be written as

$$
\begin{aligned}
& \left(A_{o}+B_{p} G\right)^{\mathrm{T}} S_{i+1}+S_{i+1}\left(A_{o}+B_{p} G\right) \\
& \quad-\left(B_{p}^{\mathrm{T}} S_{i+1}+R_{i} G\right)^{\mathrm{T}} R_{i}^{-1}\left(B_{p}^{\mathrm{T}} S_{i+1}+R_{i} G\right)+G^{\mathrm{T}} R_{i} G+C_{p}^{\mathrm{T}} C_{p}=0 .
\end{aligned}
$$


Comparing the Eqs. (4.6) and (4.7) yields $S_{i+1} \leq \bar{S}$ for $i \geq 0$. Hence it follows from Lemma 3 that $S \triangleq \lim _{i \rightarrow \infty} S_{i}$ exists and is positive definite, which is the solution to the Riccati equation (4.1).

Using Lemma 2 to compare (3.3) and (4.1) we have $Y_{p}<S$.

THEOREM 2 The minimum output variance control problem has a solution if and only if Algorithm II converges.

Proof Sufficiency Suppose that Algorithm II converges to a limit $Y_{p}=\lim _{i \rightarrow \infty} Y_{p}^{i}>0$. Then according to Theorem 1, the minimum output variance control problem has solution (3.4).

Necessity Suppose that the minimum output variance control problem has a solution. It follows from Theorem 1 that Riccati-like equation (3.3) has a solution $Y_{p}>0$. From Lemma 4 we know there exists a solution $S>0$ to Eq. (4.1). Hence Algorithm I converges to $\lim _{i \rightarrow \infty} S_{i}=S$. We now consider Algorithm II. Notice that the $\left\{Y_{p}^{i}: i=1,2, \ldots\right\}$ is monotonic nonincreasing sequence. It follows from Lemma 3 that it suffices to show that this sequence is bounded below. First, notice that $Y_{p}^{0}=S>Y_{p}$ and hence it follows, by comparing Eq. (3.3) with Eq. (4.4), that $Y_{p}^{1}>Y_{p}$. By induction, it is straightforward to show that $Y_{p}^{i}>Y_{p}$ for all $i \geq 0$. Using Lemma 3, we conclude that $\lim _{i \rightarrow \infty} Y_{p}^{i}=Y_{p}$.

\section{NUMERICAL EXAMPLE}

Consider the experimental setup in [19]. The system model is given by

$$
\begin{gathered}
A_{p}= \\
{\left[\begin{array}{cccccc}
0 & 0 & 0 & 0.0010 & 0 & 0 \\
0 & 0 & 0 & 0 & 0.0010 & 0 \\
0 & 0 & 0 & 0 & 0 & 0.0010 \\
-2.7945 & 1.6732 & -0.3762 & -0.0004 & 0.0001 & -0.0001 \\
1.6732 & -3.0804 & 1.6561 & 0.0001 & -0.0005 & 0.0000 \\
-0.3762 & 1.6561 & -1.3593 & -0.0001 & 0.0000 & -0.0004
\end{array}\right] 1.0 \mathrm{e}+03,} \\
B_{p}=\left[\begin{array}{ccccccc}
0 & 0 & 0 & -0.1786 & 0 & 0
\end{array}\right]^{\mathrm{T}}, \quad C_{p}=\left[\begin{array}{llll}
I_{3} & 0 & 0_{3}
\end{array}\right], \\
D_{p}=\left[\begin{array}{llllll}
0 & 0 & 0 & -1 & -1 & -1
\end{array}\right]^{\mathrm{T}} .
\end{gathered}
$$


In this paper, the intensity of ground acceleration is assumed to be $W_{p 1}=1$. The intensity sum $W_{\text {auc }}=W_{a 1}+W_{u 1}+W_{c 1}=0.1$. The NSR of the plant $\sigma_{p}^{2}=0.1$.

Then one can compute that the open loop output variance $J_{\text {open }}=0.0193$.

\section{FSN Controller Design}

Let $\sigma^{2}=\sigma_{a}^{2}+\sigma_{c}^{2}+\sigma_{s}^{2}+\sigma_{z}^{2}+\sigma_{u}^{2}=0.02$, i.e., the information quality $I_{Q}=50$. Set the numerical tolerance $\epsilon=1 \mathrm{e}-5$. To obtain the solution of the Riccati-like equation (3.3), we ran Algorithm I (choose $R=I_{2}$ ) and Algorithm II. The first algorithm converges in 3 iterations and the second one converges in 17 iterations. Finally, we get the minimum output variance controller

$$
G=1.0 \mathrm{e}+03\left[\begin{array}{llllll}
7.0976 & -0.5161 & 1.9501 & 0.2800 & 0.2785 & 0.3117
\end{array}\right],
$$

the minimum output variance $J_{y}=6.0681 \mathrm{e}-04$ and the control variance $J_{u}=3.0138 \mathrm{e}+04$.

\section{Information Quality $I_{Q}$ Study}

When we design an optimal controller for a given system, we might be interested in the relations among $I_{Q}, J_{y}$ and $J_{u}$, since the relations can give us suggestions for choosing hardware devices. For the system given above, we let $I_{Q}$ vary from 0.05 to 25 . The relations are given in Fig. 2. The solid line represents the 3-dimensional relation among the three quantities. The dotted lines represent the three projections. To design an optimal controller satisfying the specified performance, one can easily obtain the corresponding information quality $I_{Q}$. Then one should choose the hardware devices with appropriate quality.

\section{CONCLUSIONS}

The minimum output variance control problem is formulated and solved for finite signal-to-noise ratio models. Specifically, the necessity 


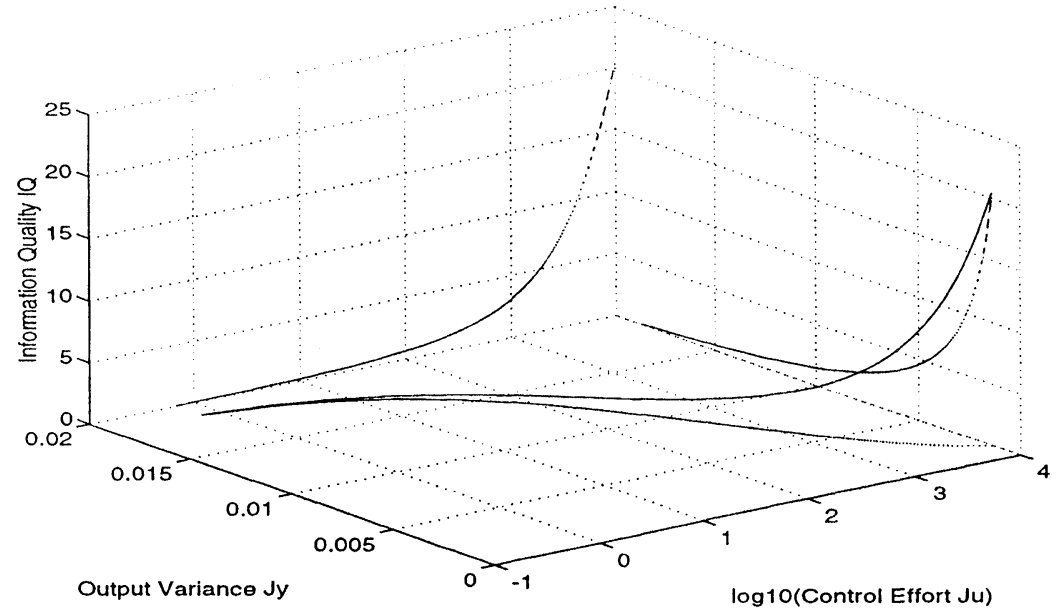

FIGURE 2 Information quality study.

and sufficiency conditions for optimality are derived. An optimal control law which involves a positive definite solution of a Riccati-like equation is derived. A numerical algorithm for solving the Riccati-like equation is given and its convergence is guaranteed if the solution exists. One special feature of the FSN optimal controller is that it achieves the maximal accuracy with finite control effort. An optimal relation among the information quality $I_{Q}$, the maximal accuracy $J_{y}$ and the minimal control effort $J_{u}$ is obtained, which provides some suggestions for choosing hardware devices.

\section{References}

[1] R.E. Skelton. Robust control of aerospace systems. In IFAC Symposium on Robust Control Design, pp. 24-32, Rio De Janeiro, Brasil, September 1994.

[2] Guojun Shi and Robert E. Skelton. State feedback covariance control for linear finite signal-to-noise ratio models. In Proc. 34th IEEE Conf. Decis. and Contr., New Orleans, Louisiana, December 1995.

[3] Uffe H. Thygesen and Robert E. Skelton. Linear systems with finite signal-to-noise ratios: A robustness approach. In Proc. 34th IEEE Conf. Decis. and Contr., New Orleans, Louisiana, December 1995.

[4] Robert E. Skelton and Guojun Shi. Finite signal-to-noise ratio models: covariance control by state feedback. In 1995 European Contr. Conf., Rome, Italy, September 1995. 
[5] C. Hsieh, R.E. Skelton and F.M. Damra. Minimum energy controllers with inequality constraints on output variances. Optimal Control Applications and Methods, 10: 347-366, 1989.

[6] Guoming Zhu, Mario A. Rotea and Robert E. Skelton. A convergent feasible algorithm for the output covariance constraint problem. In 1993 American Contr. Conf., pp. 1675-1679, San Francisco, CA, June 1993.

[7] Michel Gevers and Gang Li. Parameterizations in Control, Estimation and Filtering Problems. Springer-Verlag, New York, 1993.

[8] Ketao Liu, Robert E. Skelton and Karolos Grigoriadis. Optimal controllers for finite wordlength implementation. IEEE Trans. Automat. Contr., AC-37(9): 1294$1304 ; 1992$.

[9] R.E. Skelton. Model error concepts in control design. Int. J. Contr., 49(5): 1725-1753, 1989.

[10] D.L. Kleinman. Optimal stationary control of linear systems with controldependent noise. IEEE Trans. Automat. Contr., AC-14(6): 673-677, 1969.

[11] P.J. Mclane. Optimal stochastic control of linear systems with state- and controldependent disturbances. IEEE Trans. Automat. Contr., AC-16(6): 793-798, 1971.

[12] A.C.M. Ran and R. Vreugdenhil. Existence and comparison theorems for algebraic riccati equations for continuous- and discrete-time systems. Linear Algebra and Its Applications, 99: 63-83, 1988.

[13] L.V. Kantorovich and G.P. Akilov. Functional Analysis in Normed Spaces. Pergamon Press, New York, 1964.

[14] D.L. Kleinman. On the stability of linear stochastic systems. IEEE Trans. Automat. Contr., AC-14(3): 429-430, 1969.

[15] Robert E. Skelton. Dynamic Systems Control. John Wiley \& Sons, Inc., New York, 1988.

[16] Kemin Zhou, John Doyle and Keith Glover. Robust and Optimal Control. PrenticeHall Inc., Englewood Cliffs, NJ, 1996.

[17] D.L. Kleinman. Numerical solution of the state dependent noise problem. IEEE Trans. Automat. Contr., AC-21(6): 419-420, 1976.

[18] B.D.O. Anderson and J.B. Moore. Linear Optimal Control. Prentice-Hall Inc., Englewood Cliffs, New Jersey, 1971.

[19] L.L. Chung, R.C. Lin, T.T. Soong and A.M. Reinhorn. Experimental study of active control for MDOF seimic structure. J. Engineering Mechanics, 115(8): 1609-1627, 1989. 


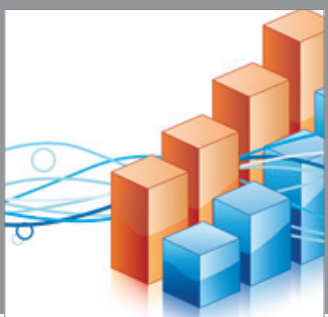

Advances in

Operations Research

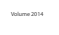

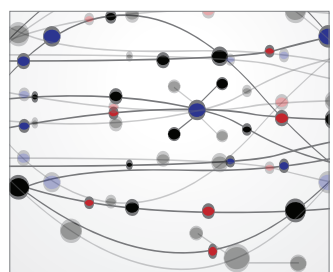

\section{The Scientific} World Journal
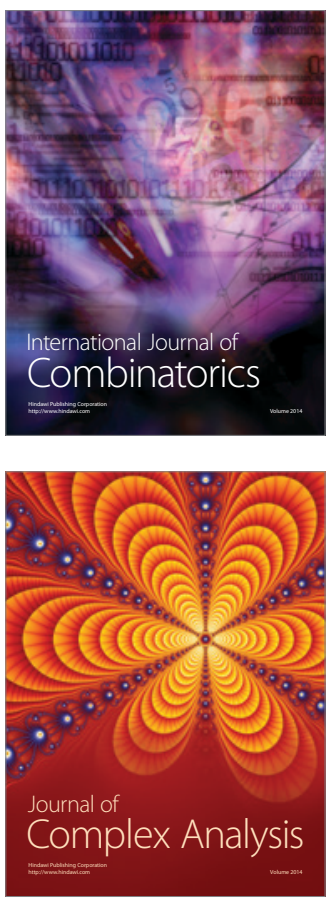

International Journal of

Mathematics and

Mathematical

Sciences
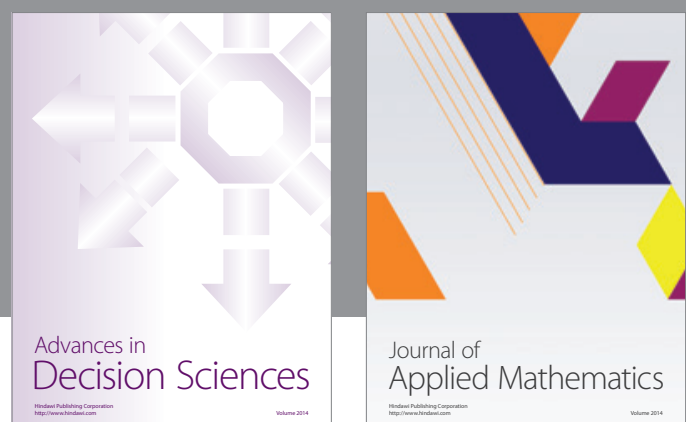

Journal of

Applied Mathematics
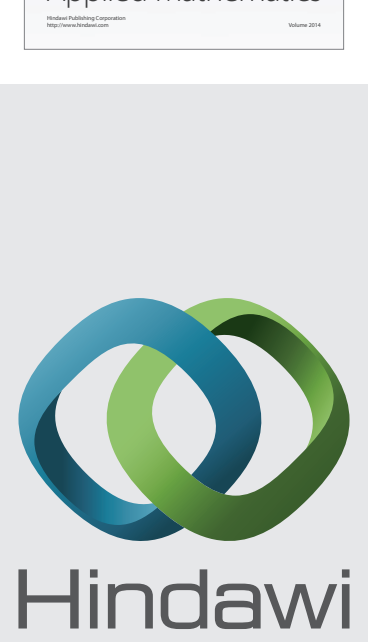

Submit your manuscripts at http://www.hindawi.com
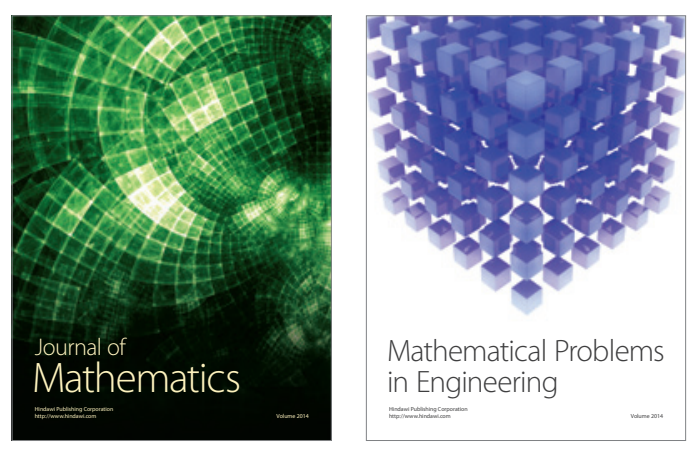

Mathematical Problems in Engineering
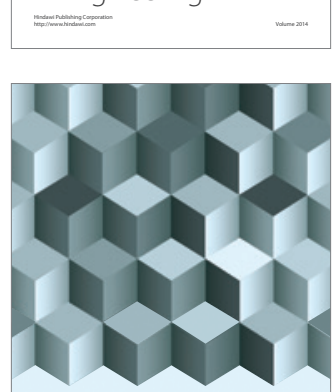

Journal of

Function Spaces
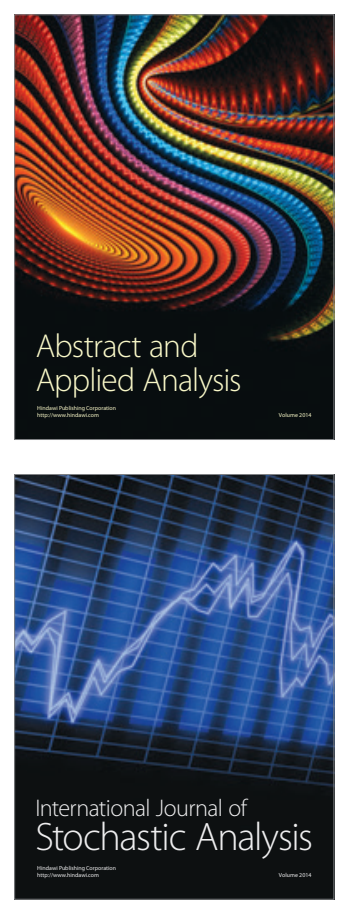

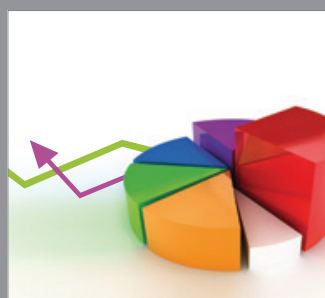

ournal of

Probability and Statistics

Promensencen
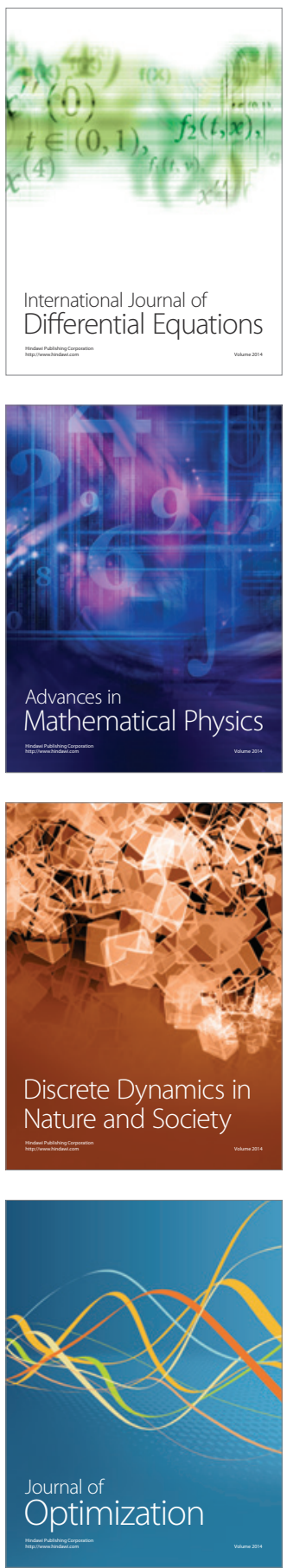